GEOMETRY IN NONLINEAR CONTROL

AND DIFFERENTIAL INCLUSIONS

BANACH CENTER PUBLICATIONS, VOLUME 32

INSTITUTE OF MATHEMATICS

POLISH ACADEMY OF SCIENCES

WARSZAWA 1995

\title{
DIFFERENTIAL FLATNESS AND DEFECT: AN OVERVIEW
}

\author{
MICHEL FLIESS \\ Laboratoire des Signaux et Systèmes, CNRS-ESE \\ Plateau de Moulon, 91192 Gif-sur-Yvette Cedex, France \\ E-mail: fliess@lss.supelec.fr \\ JEAN LÉVINE \\ Centre Automatique et Systèmes, École des Mines de Paris \\ 35, rue Saint-Honoré, 7r305 Fontainebleau Cedex, France \\ E-mail:levine@cas.ensmp.fr \\ PHILIPPE MARTIN \\ Centre Automatique et Systèmes, École des Mines de Paris \\ 35, rue Saint-Honoré, 77305 Fontainebleau Cedex, France \\ E-mail: martin@cas.ensmp.fr \\ PIERRE ROUCHON \\ Centre Automatique et Systèmes, École des Mines de Paris \\ 60, bd. Saint-Michel, 75272 Paris Cedex 06, France \\ E-mail: rouchon@cas.ensmp.fr
}

\begin{abstract}
We introduce flat systems, which are equivalent to linear ones via a special type of feedback called endogenous. Their physical properties are subsumed by a linearizing output and they might be regarded as providing another nonlinear extension of Kalman's controllability. The distance to flatness is measured by a non-negative integer, the defect. We utilize differential algebra which suits well to the fact that, in accordance with Willems' standpoint, flatness and defect are best defined without distinguishing between input, state, output and other variables. We treat an example of non-flat system, the variable-length pendulum. A high frequency control strategy is proposed such that the averaged system becomes flat.
\end{abstract}

1991 Mathematics Subject Classification: 12H05, 34C29, 93B18.

This work was partially supported by the G.R. "Automatique" of the CNRS and by the D.R.E.D. of the "Ministère de l'Éducation Nationale".

The paper is in final form and no version of it will be published elsewhere. 
1. Introduction. Our original motivation is dynamic feedback linearization which, as opposed to the static one, has only been investigated by few authors $[32,34,6,7,51]$, and remains widely open. Our point of view will be probably best explained by the following calculations where all vector fields and functions are real-analytic.

Consider

$$
\dot{x}=f(x, u) \quad\left(x \in \mathbb{R}^{n}, u \in \mathbb{R}^{m}\right),
$$

where $f(0,0)=0$ and $\operatorname{rank} \frac{\partial f}{\partial u}(0,0)=m$. The dynamic feedback linearizability of (1) means, according to [6], the existence of

1) a regular dynamic compensator

$$
\left\{\begin{array}{l}
\dot{z}=a(x, z, v) \\
u=b(x, z, v)
\end{array} \quad\left(z \in \mathbb{R}^{q}, v \in \mathbb{R}^{m}\right)\right.
$$

where $a(0,0,0)=0, b(0,0,0)=0$. The regularity assumption implies the invertibility $\left({ }^{1}\right)$ of system (2) with input $v$ and output $u$.

2) a diffeomorphism

$$
\xi=\Xi(x, z) \quad\left(\xi \in \mathbb{R}^{n+q}\right)
$$

such that (1) and (2), whose $(n+q)$-dimensional dynamics is given by

$$
\left\{\begin{array}{l}
\dot{x}=f(x, b(x, z, v)) \\
\dot{z}=a(x, z, v)
\end{array}\right.
$$

becomes, according to (3), a constant linear controllable system $\dot{\xi}=F \xi+G v$.

Up to a static state feedback and a linear invertible change of coordinates, this linear system may be written in Brunovský canonical form (see, e.g., [40]),

$$
\left\{\begin{array}{c}
y_{1}^{\left(\nu_{1}\right)}=v_{1}, \\
\vdots \\
y_{m}^{\left(\nu_{m}\right)}=v_{m},
\end{array}\right.
$$

where $\nu_{1}, \ldots, \nu_{m}$ are the controllability indices. From (2) and (3) the components of $u$ and $x$ can be expressed as real-analytic functions of the components of $y=\left(y_{1}, \ldots, y_{m}\right)$ and a finite number of their derivatives:

$$
\left\{\begin{array}{l}
x=\mathcal{A}\left(y, \dot{y}, \ldots, y^{(\alpha)}\right) \\
u=\mathcal{B}\left(y, \dot{y}, \ldots, y^{(\alpha)}\right) .
\end{array}\right.
$$

The dynamic feedback (2) is said to be endogenous if, and only if, the converse holds, i.e., if, and only if, any component of $y$ can be expressed as a real-analytic function of $x, u$ and a finite number of its derivatives:

$$
y=\mathcal{C}\left(x, u, \dot{u}, \ldots, u^{(\gamma)}\right) .
$$

$\left({ }^{1}\right)$ See $[43]$ for a definition of this concept via the structure algorithm. See $[12,11]$ for a connection with the differential algebraic approach. 
A dynamics (1) which is linearizable via such an endogenous feedback is said to be (differentially) flat; $y$, which might be regarded as a fictitious output, is called a linearizing or flat output. The terminology flat is due to the fact that $y$ plays a somehow analogous role to the flat coordinates in the differential geometric approach to the Frobenius theorem (see, e.g., [33, 46]). Notice that the use of a linearizing output was already known in the context of static state feedback (see [8] and [33, page 156]). Thanks to endogenous feedback, like quasi-static ones in a slightly different context [11], the computation of the state and input do not require the integration of any differential equation. This general idea can be traced back to works by D. Hilbert [31] and E. Cartan [4] on under-determined systems of differential equations, where the number of equations is strictly less than the number of unknowns.

Flatness is best defined by not distinguishing between input, state, output and other variables. The equations moreover might be implicit. This standpoint, which matches well with Willems' approach [56], is here taken into account by utilizing differential algebra which has already helped clarifying several questions in control theory (see, e.g., $[13,14,15,16,19]$ ).

Flatness might be seen as another nonlinear extension of Kalman's controllability. Such an assertion is surprising when having in mind the vast literature on this subject (see $[33,46]$ and the references therein). Remember, however, Willems' trajectory characterization [56] of linear controllability which can be interpreted as the freeness of the module associated to a linear system [18]. A linearizing output now is the nonlinear analogue of a basis of this free module.

We know from [6] that any single-input dynamics which is linearizable by a dynamic feedback is also linearizable by a static one. This implies the existence of non-flat systems which verify the strong accessibility property [54]. We introduce a non-negative integer, the defect, which measures the distance from flatness.

The paper is organized as follows. After some differential algebraic preliminaries, we define equivalence by endogenous feedback, flatness and defect. Their implications for uncontrolled dynamics and linear systems are examined. We discuss the link between flatness and controllability. In order to verify that some systems are not linearizable by dynamic feedback, we demonstrate a necessary condition of flatness, which is of geometric nature. We discuss the variable-length pendulum, already examined in [3]. An appropriate high-frequency control permits the approximation of this non-flat system by a flat one.

The material of this note is borrowed from [22, 23, 21, 27], where several other applications, such as motion planning, are considered. See [26] for a complete version.

2. The algebraic framework. We start with a brief review of differential fields (see also [16]) and refer to the books of Ritt [49] and Kolchin [41] and Seidenberg's paper [50] for details. Basics on the customary (non-differential) field theory may be found in the textbook by Jacobson [35] and Winter [57] (see 
also $[16,19])$; they will not be repeated here.

2.1. Basics on differential fields. An (ordinary) differential ring $R$ is a commutative ring equipped with a single derivation $\frac{d}{d t}=$ "'" such that

$$
\begin{gathered}
\forall a \in R, \quad \dot{a}=\frac{d a}{d t} \in R \\
\forall a, b \in R, \quad \frac{d}{d t}(a+b)=\dot{a}+\dot{b}, \quad \frac{d}{d t}(a b)=\dot{a} b+a \dot{b} .
\end{gathered}
$$

A constant $c \in R$ is an element such that $\dot{c}=0$. A ring of constants only contains constant elements. An (ordinary) differential field is an (ordinary) differential ring which is a field.

A differential field extension $L / K$ is given by two differential fields, $K$ and $L$, such that $K \subseteq L$ and such that the restriction to $K$ of the derivation of $L$ coincides with the derivation of $K$.

An element $\xi \in L$ is said to be differentially $K$-algebraic if, and only if, it satisfies an algebraic differential equation over $K$, i.e., if there exists a polynomial $\pi \in K\left[x_{0}, x_{1}, \ldots, x_{\nu}\right], \pi \neq 0$, such that $\pi\left(\xi, \dot{\xi}, \ldots, \xi^{(\nu)}\right)=0$. The extension $L / K$ is said to be differentially algebraic if, and only if, any element of $L$ is differentially $K$-algebraic.

An element $\xi \in L$ is said to be differentially $K$-transcendental if, and only if, it is not differentially $K$-algebraic. The extension $L / K$ is said to be differentially transcendental if, and only if, there exists at least one element of $L$ that is differentially $K$-transcendental.

A set $\left\{\xi_{i} \mid i \in I\right\}$ of elements in $L$ is said to be differentially $K$-algebraically independent if, and only if, the set of derivatives of any order, $\left\{\xi_{i}^{(\nu)} \mid i \in I, \nu=\right.$ $0,1,2, \ldots\}$, is $K$-algebraically independent. Such an independent set which is maximal with respect to inclusion is called a differential transcendence basis of $L / K$. Two such bases have the same cardinality, i.e., the same number of elements, which is called the differential transcendence degree of $L / K$ : it is denoted by $\operatorname{diff} \operatorname{tr} d^{0} L / K$. Notice that $L / K$ is differentially algebraic if, and only if, diff $\operatorname{tr} d^{0} L / K=0$.

TheOREM 1. For a finitely generated differential extension $L / K$, the next two properties are equivalent:

(i) $L / K$ is differentially algebraic;

(ii) the (non-differential) transcendence degree of $L / K$ is finite, i.e., $\operatorname{tr} d^{0} L / K$ $<\infty$.

More details and some examples may be found in [19].

2.2. Systems $\left({ }^{2}\right)$. Let $k$ be a given differential ground field. A system is a

$\left({ }^{2}\right)$ See also $[16,19]$. 
finitely generated differential extension $\mathcal{D} / k\left({ }^{3}\right)$. Such a definition corresponds to a finite number of quantities which are related by a finite number of algebraic differential equations over $k\left({ }^{4}\right)$. We do not distinguish in this setting between input, state, output and other types of variables. This field-theoretic language therefore fits Willems standpoint [56] on systems. The differential order of the system $\mathcal{D} / k$ is the differential transcendence degree of the extension $\mathcal{D} / k$.

Example. Set $k=\mathbb{R} ; \mathcal{D} / k$ is the differential field generated by the four unknowns $x_{1}, x_{2}, x_{3}, x_{4}$ related by the two algebraic differential equations:

$$
\dot{x}_{1}+\ddot{x}_{3} \dot{x}_{4}=0, \quad \dot{x}_{2}+\left(x_{1}+\ddot{x}_{3} x_{4}\right) x_{4}=0 .
$$

Clearly, diff $\operatorname{tr} d^{0} \mathcal{D} / k=2$ : it is equal to the number of unknowns minus the number of equations.

Denote by $k\langle u\rangle$ the differential field generated by $k$ and by a finite set $u=$ $\left(u_{1}, \ldots, u_{m}\right)$ of differential $k$-indeterminates: $u_{1}, \ldots, u_{m}$ are differentially $k$-algebraically independent, i.e., diff $\operatorname{tr} d^{0} k\langle u\rangle / k=m$. A dynamics with (independent) input $u$ is a finitely generated differentially algebraic extension $\mathcal{D} / k\langle u\rangle$. Note that the number $m$ of independent input channels is equal to the differential order of the corresponding system $\mathcal{D} / k$. An output $y=\left(y_{1}, \ldots, y_{p}\right)$ is a finite set of differential quantities in $\mathcal{D}$.

According to theorem 1 , there exists a finite transcendence basis $x=\left(x_{1}, \ldots\right.$ $\left.\ldots, x_{n}\right)$ of $\mathcal{D} / k\langle u\rangle$. Consequently, any component of $\dot{x}=\left(\dot{x}_{1}, \ldots, \dot{x}_{n}\right)$ and of $y$ is $k\langle u\rangle$-algebraically dependent on $x$, which plays the role of a (generalized) state. This yields:

$$
\left\{\begin{array}{l}
A_{1}\left(\dot{x}_{1}, x, u, \dot{u}, \ldots, u^{\left(\alpha_{1}\right)}\right)=0 \\
\vdots \\
A_{n}\left(\dot{x}_{n}, x, u, \dot{u}, \ldots, u^{\left(\alpha_{n}\right)}\right)=0 \\
B_{1}\left(y_{1}, x, u, \dot{u}, \ldots, u^{\left(\beta_{1}\right)}\right)=0 \\
\vdots \\
B_{p}\left(y_{p}, x, u, \dot{u}, \ldots, u^{\left(\beta_{p}\right)}\right)=0
\end{array}\right.
$$

where the $A_{i}$ 's and $B_{j}$ 's are polynomial over $k$. The integer $n$ is the dimension of the dynamics $\mathcal{D} / k\langle u\rangle$. We refer to $[20,28]$ for a discussion of such generalized state-variable representations (5) and their relevance to practice.

$\left({ }^{3}\right)$ Two systems $\mathcal{D} / k$ and $\widetilde{\mathcal{D}} / k$ are, of course, identified if, and only if, there exists a differential $k$-isomorphism between them (a differential $k$-isomorphism commutes with $d / d t$ and preserves every element of $k$ ).

$\left({ }^{4}\right)$ It is a standard fact in classic commutative algebra and algebraic geometry (c.f. [30]) that one needs prime ideals for interpreting "concrete" equations in the language of field theory. In our differential setting, we of course need differential prime ideals (see [41] and also [19] for an elementary exposition). For the verification of the prime character of the differential ideals see [14, lemma 5.2, page 158] and [26]. 
EXAmple (continued). Set $u_{1}=x_{3}$ and $u_{2}=\dot{x}_{4}$. The extension $\mathcal{D} / \mathbb{R}\langle u\rangle$ is differentially algebraic and yields the representation

$$
\left\{\begin{array}{l}
\dot{x}_{1}=-\ddot{u}_{1} u_{2}, \\
\dot{x}_{2}=-\left(x_{1}+\ddot{u}_{1} x_{4}\right) x_{4}, \\
\dot{x}_{4}=u_{2} .
\end{array}\right.
$$

The dimension of the dynamics is 3 and $\left(x_{1}, x_{2}, x_{4}\right)$ is a generalized state. It would be 5 if we set $u_{1}=\ddot{x}_{3}$ and $u_{2}=\dot{x}_{4}$, and the corresponding representation becomes causal in the classical sense.

2.3. Modules and linear systems $\left({ }^{5}\right)$. Let $k$ be again a given differential ground field. Denote by $k\left[\frac{d}{d t}\right]$ the ring of linear differential operators of the type

$$
\sum_{\text {finite }} a_{\alpha} \frac{d^{\alpha}}{d t^{\alpha}} \quad\left(a_{\alpha} \in k\right)
$$

This ring is commutative if, and only if, $k$ is a field of constants. Nevertheless, in the general non-commutative case, $k\left[\frac{d}{d t}\right]$ still is a principal ideal ring and the most important properties of left $k\left[\frac{d}{d t}\right]$-modules mimic those of modules over commutative principal ideal rings (see $[9]$ ).

Let $M$ be a left $k\left[\frac{d}{d t}\right]$-module. An element $m \in M$ is said to be torsion if, and only if, there exists $\pi\left[\frac{d}{d t}\right], \pi \neq 0$, such that $\pi \cdot m=0$. The set of all torsion elements of $M$ is a submodule $T$, which is called the torsion submodule of $M$. The module $M$ is said to be torsion if, and only if, $M=T$. The following result can be regarded as the linear counterpart of theorem 1 .

Proposition 1. For a finitely generated left $k\left[\frac{d}{d t}\right]$-module $M$, the next two properties are equivalent:

(i) $M$ is torsion;

(ii) the dimension of $M$ as a $k$-vector space is finite.

A finitely generated module $M$ is free if, and only if, its torsion submodule $T$ is trivial, i.e., $T=\{0\}\left({ }^{6}\right)$. Any finitely generated module $M$ can be written $M=T \oplus \Phi$ where $T$ is the torsion submodule of $M$ and $\Phi$ is a free module. The rank of $M$, denoted by $\operatorname{rk} M$, is the cardinality of any basis of $\Phi$. Thus, $M$ is torsion if, and only if, $\mathrm{rk} M=0$.

A linear system is, by definition, a finitely generated left $k\left[\frac{d}{d t}\right]$-module $\Lambda$. We are thus dealing with a finite number of variables which are related by a finite number of linear homogeneous differential equations and our setting appears to be strongly related to Willems' approach [56]. The differential order of $\Lambda$ is the rank of $\Lambda$.

$\left({ }^{5}\right)$ See also [17].

$\left({ }^{6}\right)$ This is not the usual definition of free modules, but a characterization which holds holds for finitely generated modules over principal ideal rings, where any torsion free module is free (see [9]). 
A linear dynamics with input $u=\left(u_{1}, \ldots, u_{m}\right)$ is a linear system $\Lambda$ which contains $u$ such that the quotient module $\Lambda /[u]$ is torsion, where $[u]$ denotes the left $k\left[\frac{d}{d t}\right]$-module spanned by the components of $u$. The input is assumed to be independent, i.e., the module $[u]$ is free. This implies that the differential order of $\Lambda$ is equal to $m$. A classical Kalman state variable representation is always possible:

$$
\frac{d}{d t}\left(\begin{array}{c}
x_{1} \\
\vdots \\
x_{n}
\end{array}\right)=A\left(\begin{array}{c}
x_{1} \\
\vdots \\
x_{n}
\end{array}\right)+B\left(\begin{array}{c}
u_{1} \\
\vdots \\
u_{m}
\end{array}\right)
$$

where

- the dimension $n$ of the state $x=\left(x_{1}, \ldots, x_{n}\right)$, which is called the dimension of the dynamics, is equal to the dimension of the torsion module $\Lambda /[u]$ as a $k$-vector space,

- the matrices $A$ and $B$, of appropriate sizes, have their entries in $k$.

An output $y=\left(y_{1}, \ldots, y_{p}\right)$ is a set of elements in $\Lambda$. It leads to the following output map:

$$
\left(\begin{array}{c}
y_{1} \\
\vdots \\
y_{p}
\end{array}\right)=C\left(\begin{array}{c}
x_{1} \\
\vdots \\
x_{n}
\end{array}\right)+\sum_{\text {finite }} D_{\nu} \frac{d^{\nu}}{d t^{\nu}}\left(\begin{array}{c}
u_{1} \\
\vdots \\
u_{m}
\end{array}\right) .
$$

The controllability of (7) can be expressed in a module-theoretical language which is independent of any denomination of variables. Controllability is equivalent to the freeness of the module 4 . This just is an algebraic counterpart [18] of Willems' trajectory characterization [56]. When the system is uncontrollable, the torsion submodule corresponds to the Kalman uncontrollability subspace.

R e mark 1. The relationship with the general differential field setting is obtained by producing a formal multiplication. The symmetric tensor product [35] of a linear system $\Lambda$, where $\Lambda$ is viewed as a $k$-vector space, is an integral differential ring. Its quotient field $\mathcal{D}$, which is a differential field, corresponds to the nonlinear field theoretic description of linear systems.

2.4. Differentials and tangent linear systems. Differential calculus, which plays such a role in analysis and in differential geometry, admits a nice analogue in commutative algebra $[41,57]$ which has been extended to differential algebra by Johnson [38].

To a finitely generated differential extension $L / K$, associate a mapping $d_{L / K}$ : $L \rightarrow \Omega_{L / K}$, called (Kähler) differential $\left({ }^{7}\right)$ and where $\Omega_{L / K}$ is a finitely generated

$\left({ }^{7}\right)$ For any $a \in L, d_{L / K} a$ should be intuitively understood, like in analysis and differential geometry, as a "small" variation of $a$. 
left $L\left[\frac{d}{d t}\right]$-module, such that

$$
\begin{array}{ll}
\forall a \in L & d_{L / K}\left(\frac{d a}{d t}\right)=\frac{d}{d t}\left(d_{L / K} a\right) \\
\forall a, b \in L & d_{L / K}(a+b)=d_{L / K} a+d_{L / K} b \\
& d_{L / K}(a b)=b d_{L / K} a+a d_{L / K} b \\
\forall c \in K & d_{L / K} c=0 .
\end{array}
$$

Elements of $K$ behave like constants with respect to $d_{L / K}$. Properties of the extension $L / K$ can be translated into the linear module-theoretic framework of $\Omega_{L / K}$ :

- A set $\xi=\left(\xi_{1}, \ldots, \xi_{m}\right)$ is a differential transcendence basis of $L / K$ if, and only if, $d_{L / K} \xi=\left(d_{L / K} \xi_{1}, \ldots, d_{L / K} \xi_{m}\right)$ is a maximal set of $L\left[\frac{d}{d t}\right]$-linearly independent elements in $\Omega_{L / K}$. Thus, diff $\operatorname{tr} d^{0} L / K=\operatorname{rk} \Omega_{L / K}$.

- The extension $L / K$ is differentially algebraic if, and only if, the module $\Omega_{L / K}$ is torsion. A set $x=\left(x_{1}, \ldots, x_{n}\right)$ is a transcendence basis of $L / K$ if, and only if, $d_{L / K} x=\left(d_{L / K} x_{1}, \ldots, d_{L / K} x_{n}\right)$ is a basis of $\Omega_{L / K}$ as $L$-vector space.

- the extension $L / K$ is algebraic if, and only if, $\Omega_{L / K}$ is trivial, i.e., $\Omega_{L / K}$ $=\{0\}$.

The tangent (or variational) linear system associated to the system $\mathcal{D} / k$ is the left $\mathcal{D}\left[\frac{d}{d t}\right]$-module $\Omega_{\mathcal{D} / k}$. To a dynamics $\mathcal{D} / k\langle u\rangle$ is associated the tangent (or variational) dynamics $\Omega_{\mathcal{D} / k}$ with the tangent (or variational) input $d_{L / K} u=$ $\left(d_{L / K} u_{1}, \ldots, d_{L / K} u_{m}\right)$. The tangent (or variational) output associated to $y=$ $\left(y_{1}, \ldots, y_{p}\right)$ is $d_{L / K} y=\left(d_{L / K} y_{1}, \ldots, d_{L / K} y_{p}\right)$.

\section{Equivalence, flatness and defect}

3.1. Equivalence of systems and endogenous feedback. Two systems $\mathcal{D} / k$ and $\widetilde{\mathcal{D}} / k$ are said to be equivalent or equivalent by endogenous feedback if, and only if, any element of $\mathcal{D}$ (resp. $\widetilde{\mathcal{D}}$ ) is algebraic over $\widetilde{\mathcal{D}}$ (resp. $\mathcal{D}){ }^{8}$ ). Two dynamics, $\mathcal{D} / k\langle u\rangle$ and $\widetilde{\mathcal{D}} / k\langle\widetilde{u}\rangle$, are said to be equivalent if, and only if, the corresponding systems, $\mathcal{D} / k$ and $\widetilde{\mathcal{D}} / k$, are.

Proposition 2. Two equivalent systems (resp. dynamics) have the same differential order, i.e., the same number of independent input channels.

Proof. Denote by $K$ the differential field generated by $\mathcal{D}$ and $\widetilde{\mathcal{D}}: K / \mathcal{D}$ and $K / \widetilde{\mathcal{D}}$ are algebraic extensions. Therefore,

$$
\operatorname{diff} \operatorname{tr} d^{0} \mathcal{D} / k=\operatorname{diff} \operatorname{tr} d^{0} K / k=\operatorname{diff} \operatorname{tr} d^{0} \widetilde{\mathcal{D}} / k .
$$

$\left({ }^{8}\right)$ According to footnote 3, this definition of equivalence can also be read as follows: two systems $\mathcal{D} / k$ and $\widetilde{\mathcal{D}} / k$ are equivalent if, and only if, there exist two algebraic extensions $\mathscr{D} / \mathcal{D}$ and $\widetilde{D} / \widetilde{\mathcal{D}}$, and a differential $k$-isomorphism $\Phi$ between $\mathscr{D} / k$ and $\widetilde{\mathscr{D}} / k$. 
Remark 2. The counterpart in differential algebraic geometry [41, 42] of the previous equivalence goes beyond the differential extension [5] of the classical birational equivalence [30], which relies on the field of isomorphisms.

Consider two equivalent dynamics, $\mathcal{D} / k\langle u\rangle$ and $\widetilde{\mathcal{D}} / k\langle\widetilde{u}\rangle$. Let $n$ (resp. $\widetilde{n}$ ) be the dimension of $\mathcal{D} / k\langle u\rangle$ (resp. $\widetilde{\mathcal{D}} / k\langle\widetilde{u}\rangle)$. In general, $n \neq \widetilde{n}$. Write

$$
A_{i}\left(\dot{x}_{i}, x, u, \dot{u}, \ldots, u^{\left(\alpha_{i}\right)}\right)=0, \quad i=1, \ldots, n,
$$

and

$$
\widetilde{A}_{i}\left(\dot{\widetilde{x}}_{i}, \widetilde{x}, \widetilde{u}, \dot{\tilde{u}}, \ldots, \widetilde{u}^{\left(\widetilde{\alpha}_{i}\right)}\right)=0, \quad i=1, \ldots, \widetilde{n}
$$

the generalized state variable representations of $\mathcal{D} / k\langle u\rangle$ and $\widetilde{\mathcal{D}} / k\langle\widetilde{u}\rangle$, respectively. The algebraicity of any element of $\mathcal{D}(\operatorname{resp} . \widetilde{\mathcal{D}})$ over $\widetilde{\mathcal{D}}(\operatorname{resp} . \mathcal{D})$ yields the following relationships between (8) and (9):

$$
\begin{array}{rlrl}
\varphi_{i}\left(u_{i}, \widetilde{x}, \widetilde{u}, \dot{\widetilde{u}}, \ldots, \widetilde{u}^{\left(\nu_{i}\right)}\right) & =0, & & i=1, \ldots, m, \\
\sigma_{\alpha}\left(x_{\alpha}, \widetilde{x}, \widetilde{u}, \dot{\widetilde{u}}, \ldots, \widetilde{u}^{\left(\mu_{\alpha}\right)}\right) & =0, & \alpha=1, \ldots, n, \\
\widetilde{\varphi}_{i}\left(\widetilde{u}_{i}, x, u, \dot{u}, \ldots, u^{\left(\widetilde{\nu}_{i}\right)}\right) & =0, & i=1, \ldots, m, \\
\widetilde{\sigma}_{\alpha}\left(\widetilde{x}_{\alpha}, x, u, \dot{u}, \ldots, u^{\left(\widetilde{\mu}_{\alpha}\right)}\right) & =0, & \alpha=1, \ldots, \widetilde{n},
\end{array}
$$

where the $\varphi_{i}$ 's, $\sigma_{\alpha}$ 's, $\widetilde{\varphi}_{i}$ 's and $\widetilde{\sigma}_{\alpha}$ 's are polynomials over $k$.

The two dynamic feedbacks corresponding to (10) are called endogenous as they do not necessitate the introduction of any variable that is transcendental over $\mathcal{D}$ and $\widetilde{\mathcal{D}}$ (see also [44]). If we know $\widetilde{x}$ (resp. $x$ ), we can calculate $u$ (resp. $\widetilde{u}$ ) from $\widetilde{u}$ (resp. $u$ ) without integrating any differential equation.

Remark 3. Take the dynamics $\mathcal{D} / k\langle u\rangle$ and a finitely generated algebraic extension $\overline{\mathcal{D}} / \mathcal{D}$. The two dynamics $\mathcal{D} / k\langle u\rangle$ and $\overline{\mathcal{D}} / k\langle u\rangle$, which are of course equivalent, have the same dimension and can be given the same state variable representation (8). In the sequel, a system $\mathcal{D} / k\langle u\rangle$ will be defined up to a finitely generated algebraic extension of $\mathcal{D}$.

Remark 4. The tangent linear systems (see subsection 2.4) of two equivalent systems are strongly related and, in fact, are "almost identical". Take two equivalent systems $\mathcal{D}_{1} / k$ and $\mathcal{D}_{2} / k$ and denote by $\mathcal{D}$ the smallest algebraic extension of $\mathcal{D}_{1}$ and $\mathcal{D}_{2}$. It is straightforward to check that the three left $\mathcal{D}\left[\frac{d}{d t}\right]$-modules $\Omega_{\mathcal{D} / k}, \mathcal{D} \otimes_{\mathcal{D}_{1}} \Omega_{\mathcal{D}_{1} / k}$ and $\mathcal{D} \otimes_{\mathcal{D}_{2}} \Omega_{\mathcal{D}_{2} / k}$ are isomorphic (see [30, 35]).

3.2. Flatness and defect. Like in the non-differential case, a differential extension $L / K$ is said to be purely differentially transcendental if, and only if, there exists a differential transcendence basis $\xi=\left\{\xi_{i} \mid i \in I\right\}$ of $L / K$ such that $L=K\langle\xi\rangle$. A system $\mathcal{D} / k$ is called purely differentially transcendental if, and only if, the extension $\mathcal{D} / k$ is so.

A system $\mathcal{D} / k$ is called (differentially) flat if, and only if, it is equivalent to a purely differentially transcendental system $L / k$. A differential transcendence 
basis $y=\left(y_{1}, \ldots, y_{m}\right)$ of $L / k$ such that $L=k\langle y\rangle$ is called a linearizing or flat output of the system $\mathcal{D} / k$.

EXAMPLE (continued). Let us prove that $y=\left(y_{1}, y_{2}\right)$ with

$$
y_{1}=x_{2}+\frac{\left(x_{1}+\ddot{x}_{3} x_{4}\right)^{2}}{2 x_{3}^{(3)}}, \quad y_{2}=x_{3},
$$

is a linearizing output for (4). Set $\sigma=x_{1}+\ddot{x}_{3} x_{4}$. Differentiating $y_{1}=x_{2}+\sigma^{2} / 2 y_{2}^{(3)}$, we have, using (4), $\sigma^{2}=-2 \dot{y}_{1}\left(y_{2}^{(3)}\right)^{2} / y_{2}^{(4)}$. Thus $x_{2}=y_{1}-\sigma^{2} /\left(2 y_{2}^{(3)}\right)$ is an algebraic function of $\left(y_{1}, \dot{y}_{1}, y_{2}^{(3)}, y_{2}^{(4)}\right)$. Since $x_{4}=-\dot{x}_{2} / \sigma$ and $x_{1}=\sigma-\ddot{y}_{2} x_{4}, x_{4}$ and $x_{1}$ are algebraic functions of $\left(y_{1}, \dot{y}_{1}, \ddot{y}_{1}, \ddot{y}_{2}, y_{2}^{(3)}, y_{2}^{(4)}, y_{2}^{(5)}\right)$. Remark there exist many other linearizing outputs such as $\widetilde{y}=\left(\widetilde{y}_{1}, \widetilde{y}_{2}\right)=\left(2 y_{1} y_{2}^{(3)}, y_{2}\right)$ with inverse transformation being $y=\left(\widetilde{y}_{1} / 2 \widetilde{y}_{2}^{(3)}, \widetilde{y}_{2}\right)$.

Take an arbitrary system $\mathcal{D} / k$ of differential order $m$. Among all the possible choices of sets $z=\left(z_{1}, \ldots, z_{m}\right)$ of $m$ differential $k$-indeterminates which are algebraic over $\mathcal{D}$, take one such that $\operatorname{tr} d^{0} \mathcal{D}\langle z\rangle / k\langle z\rangle$ is minimum, say $\delta$. This integer $\delta$ is called the defect of the system $\mathcal{D} / k$. The next result is obvious.

Proposition 3. A system $\mathcal{D} / k$ is flat if, and only if, its defect is zero.

ExAMPLE. The defect of the system generated by $x_{1}$ and $x_{2}$ satisfying $\dot{x}_{1}=$ $x_{1}+\left(\dot{x}_{2}\right)^{3}$ is one. Its general solution cannot be expressed without the integration of, at least, one differential equation.

\subsection{Basic examples}

3.3.1. Uncontrolled dynamical systems. An uncontrolled dynamical system is, in our field-theoretic language [16], a finitely generated differentially algebraic extension $\mathcal{D} / k$ : diff $\operatorname{tr} d^{0} \mathcal{D} / k=0$ implies the non-existence of any differential $k$ indeterminate algebraic over $\mathcal{D}$. Thus, the defect of $\mathcal{D} / k$ is equal to $\operatorname{tr} d^{0} \mathcal{D} / k$, i.e., to the dimension of the dynamical system $\mathcal{D} / k$, which corresponds to the state variable representation $A_{i}\left(\dot{x}_{i}, x\right)=0$, where $x=\left(x_{1}, \ldots, x_{n}\right)$ is a transcendence basis of $\mathcal{D} / k$. Flatness means that $\mathcal{D} / k$ is algebraic in the (non-differential) sense: the dynamics $\mathcal{D} / k$ is then said to be trivial.

3.3.2. Linear systems. The defect of $\Lambda$ is, by definition, the defect of its associated differential field extension $\mathcal{D} / k$ (see remark 1 ).

THEOREM 2. The defect of a linear system is equal to the dimension of its torsion submodule, i.e., to the dimension of its Kalman uncontrollable subspace. A linear system is flat if, and only if, it is controllable.

Proof. Take the decomposition $\Lambda=T \oplus \Phi$, of section 2.3 , where $T$ is the torsion submodule and $\Phi$ a free module. A basis $b=\left(b_{1}, \ldots, b_{m}\right)$ of $\Phi$ plays the role of a linearizing output when $\Lambda$ is free: the system then is flat. When $T \neq\{0\}$, the differential field extension $\mathcal{T} / k$ generated by $T$ is differentially algebraic and its 
(non-differential) transcendence degree is equal to the dimension of $T$ as $k$-vector space. The conclusion follows at once.

R e mark 5. The above arguments can be made more concrete by considering a linear dynamics over $\mathbb{R}$. If it is controllable, we may write it, up to a static feedback, in its Brunovský canonical form:

$$
y^{\left(\nu_{i}\right)}=u_{i}, \quad(i=1, \ldots, m)
$$

where the $\nu_{i}$ 's are the controllability indices and $y=\left(y_{1}, \ldots, y_{m}\right)$ is a linearizing output. In the uncontrollable case, the defect $d$ is the dimension of the uncontrollable subspace:

where $M$ is a $d \times d$ matrix over $\mathbb{R}$.

$$
\frac{d}{d t}\left(\begin{array}{c}
\xi_{1} \\
\vdots \\
\xi_{d}
\end{array}\right)=M\left(\begin{array}{c}
\xi_{1} \\
\vdots \\
\xi_{d}
\end{array}\right)
$$

3.4. A necessary condition for flatness. Consider the system $\mathcal{D} / k$ where $\mathcal{D}=$ $k\langle w\rangle$ is generated by a finite set of quantities $w=\left(w_{1}, \ldots, w_{q}\right)$ which are not necessarily differentially $k$-algebraically independent. The $w_{i}$ 's are related by a finite set, $\Xi\left(w, \dot{w}, \ldots, w^{(\nu)}\right)=0$, of algebraic differential equations. Define the algebraic variety $S$ corresponding to $\Xi\left(\xi^{0}, \ldots, \xi^{\nu}\right)=0$ in the $(\nu+1) q$-dimensional affine space with coordinates

$$
\xi^{j}=\left(\xi_{1}^{j}, \ldots, \xi_{q}^{j}\right), \quad j=0,1, \ldots, \nu .
$$

THEOREM 3. If the system $\mathcal{D} / k$ is flat, the affine algebraic variety $S$ contains at each regular point a straight line parallel to the $\xi^{\nu}$-axes.

Proof. The components of $w, \dot{w}, \ldots, w^{(\nu-1)}$ are algebraically dependent on the components of a linearizing output $y=\left(y_{1}, \ldots, y_{m}\right)$ and a finite number of their derivatives. Let $\mu$ be the highest order of these derivatives. The components of $w^{(\nu)}$ depend linearly on the components of $y^{(\mu+1)}$, which play the role of independent parameters for the coordinates $\xi_{1}^{\nu}, \ldots, \xi_{q}^{\nu}$.

The above condition is not sufficient. Consider the system $\mathcal{D} / \mathbb{R}$ generated by $\left(x_{1}, x_{2}, x_{3}\right)$ satisfying $\dot{x}_{1}=\left(\dot{x}_{2}\right)^{2}+\left(\dot{x}_{3}\right)^{3}$. This system does not satisfy the necessary condition: it is not flat. The same system $\mathcal{D}$ can be defined in another way by setting $x_{4}=\dot{x}_{2}$ : then, $\mathcal{D}$ is generated by the quantities $\left(x_{1}, x_{2}, x_{3}, x_{4}\right)$ related by $\dot{x}_{1}=\left(x_{4}\right)^{2}+\left(\dot{x}_{3}\right)^{3}$ and $x_{4}=\dot{x}_{2}$. Those new equations now satisfy our necessary criterion.

3.5. Flatness and controllability. Sussmann and Jurdjevic [54] have introduced in the differential geometric setting the concept of strong accessibility for dynamics of the form $\dot{x}=f(x, u)$. Sontag [52] showed that strong accessibility implies the existence of controls such that the linearized system around a trajectory passing through a point $a$ of the state-space is controllable. Coron [10] and Sontag [53] demonstrated that, for any $a$, those controls are generic. 
The above considerations with those of section 2.3 and 2.4 lead in our context to the following definition of controllability, which is independent of any distinction between variables: a system $\mathcal{D} / k$ is said to be controllable (or strongly accessible) if, and only if, its tangent linear system is controllable, i.e., if, and only if, the module $\Omega_{\mathcal{D} / k}$ is free.

Remark 4 shows that this definition is invariant under our equivalence via endogenous feedback.

Proposition 4. A flat system is controllable.

P r o o f. It suffices to prove it for a purely differentially transcendental extensions $k\langle y\rangle / k$, where $y=\left(y_{1}, \ldots, y_{m}\right)$. The module $\Omega_{k\langle y\rangle / k}$, which is spanned by $d_{k\langle y\rangle / k} y_{1}, \ldots, d_{k\langle y\rangle / k} y_{m}$, is necessarily free.

The converse is false as demonstrated by numerous examples of strongly accessible single-input dynamics $\dot{x}=f(x, u)$ which are not linearizable by static feedback and therefore neither by dynamic ones [6].

Flatness which is equivalent to the possibility of expressing any element of the system as a function of the linearizing output and a finite number of its derivatives, may be viewed as the nonlinear extension of linear controllability, if the latter is characterized by free modules. Whereas the strong accessibility property only is an "infinitesimal" generalization of linear controllability, flatness should be viewed as a more tractable one. This will be enhanced in section 4 where controllable systems of nonzero defect are treated using high-frequency control that enables to approximate them by flat systems for which the control design is straightforward.

4. An example: high-frequency control of non-flat systems. A whole set of examples may be found in [26] including flat as well as non-flat systems. We have chosen here a non-flat variable length pendulum, which has been treated in [3] by optimal control. We are introducing a method for controlling of a non-flat system via its approximation by an averaged one. For the use of high-frequency control in different contexts see also $[1,2,45,55]$. The notations are summarized on figure 1 . We assume as in [3] that the velocity $\dot{u}=v$ is the control. The

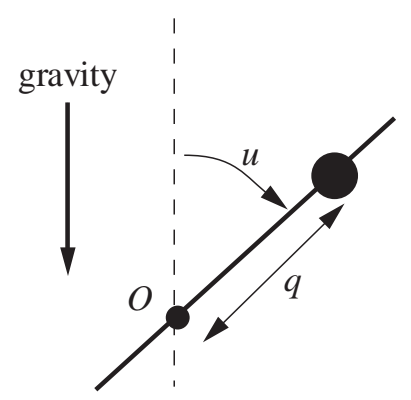

Fig. 1. Pendulum with variable length 
equations of motion are:

$$
\left\{\begin{array}{l}
\dot{q}=p, \\
\dot{p}=-\cos u+q v^{2}, \\
\dot{u}=v,
\end{array}\right.
$$

where mass and gravity are normalized to 1.

R e mark 6. Using $\tan (u / 2)$ instead of $u$, lead to differential-algebraic equations. The prime character of the corresponding differential ideal may be easily verified by utilizing [14].

This system is not flat since it admits only one control variable and is not linearizable via static feedback [6]. It is, however, strongly accessible.

Set

$$
v=v_{1}+v_{2} \cos (t / \varepsilon)
$$

where $v_{1}$ and $v_{2}$ are auxiliary controls, $0<\varepsilon \ll 1$. It is then natural to consider the following averaged control system:

$$
\left\{\begin{array}{l}
\dot{\bar{q}}=\bar{p}, \\
\dot{\bar{p}}=-\cos \bar{u}+q\left(v_{1}\right)^{2}+q\left(v_{2}\right)^{2} / 2, \\
\dot{\bar{u}}=v_{1} .
\end{array}\right.
$$

It admits two control variables, $v_{1}$ and $v_{2}$, whereas the original system (11) admits only one, $v$. Moreover (12) is flat with $(\bar{q}, \bar{u})$ as linearizing output.

The static feedback

$$
v_{1}=w_{1}, \quad v_{2}=\sqrt{2\left(\frac{w_{2}+\cos \bar{u}}{\bar{q}}-\left(w_{1}\right)^{2}\right)},
$$

transforms (12) into

$$
\dot{\bar{u}}=w_{1}, \quad \ddot{\bar{q}}=w_{2} .
$$

Set

$$
\begin{aligned}
& w_{1}=-\frac{\bar{u}-u^{s p}}{\tau_{1}} \\
& w_{2}=-\left(\frac{1}{\tau_{1}}+\frac{1}{\tau_{2}}\right) \bar{p}-\frac{1}{\tau_{1} \tau_{2}}\left(\bar{q}-q^{s p}\right)
\end{aligned}
$$

with $\left.\tau_{1}, \tau_{2}>0, u^{s p} \in\right]-\pi / 2, \pi / 2\left[, q^{s p}>0\right.$. The closed-loop averaged system (12, $13,15)$ admits an hyperbolic equilibrium point $\left(u^{s p}, q^{s p}\right)$, which is asymptotically stable.

Consider now (11) and the high-frequency control $v=v_{1}+v_{2} \sin (t / \varepsilon), 0<\varepsilon \ll$ $1 ;\left(v_{1}, v_{2}\right)$ is given by $(13,15)$ where $\bar{q}, \bar{p}$ and $\bar{u}$ are replaced by $q, p$ and $u$. Then, the corresponding averaged system is nothing else but (14) with $w_{1}$ and $w_{2}$ given by (15). Since the averaged system admits a hyperbolic asymptotically stable equilibrium, the perturbed system admits a hyperbolic asymptotically stable limit cycle around $(q, p, u)=\left(q^{s p}, 0, u^{s p}\right)[29$, theorem 4.1.1, page 168]: such control 
maintains $(u, q)$ near $\left(u^{s p}, q^{s p}\right)$. Moreover this control method is robust in the following sense: the existence and the stability of the limit cycle is not destroyed by small static errors in the measurements of $q, p$ and $u$.

As illustrated by the simulations of figure 2 , the generalization to trajectory tracking for $q$ and $u$ is straightforward. These simulations give the influence of $\varepsilon$. The design control parameters are $\tau_{1}=0.5, \tau_{2}=0.4$.
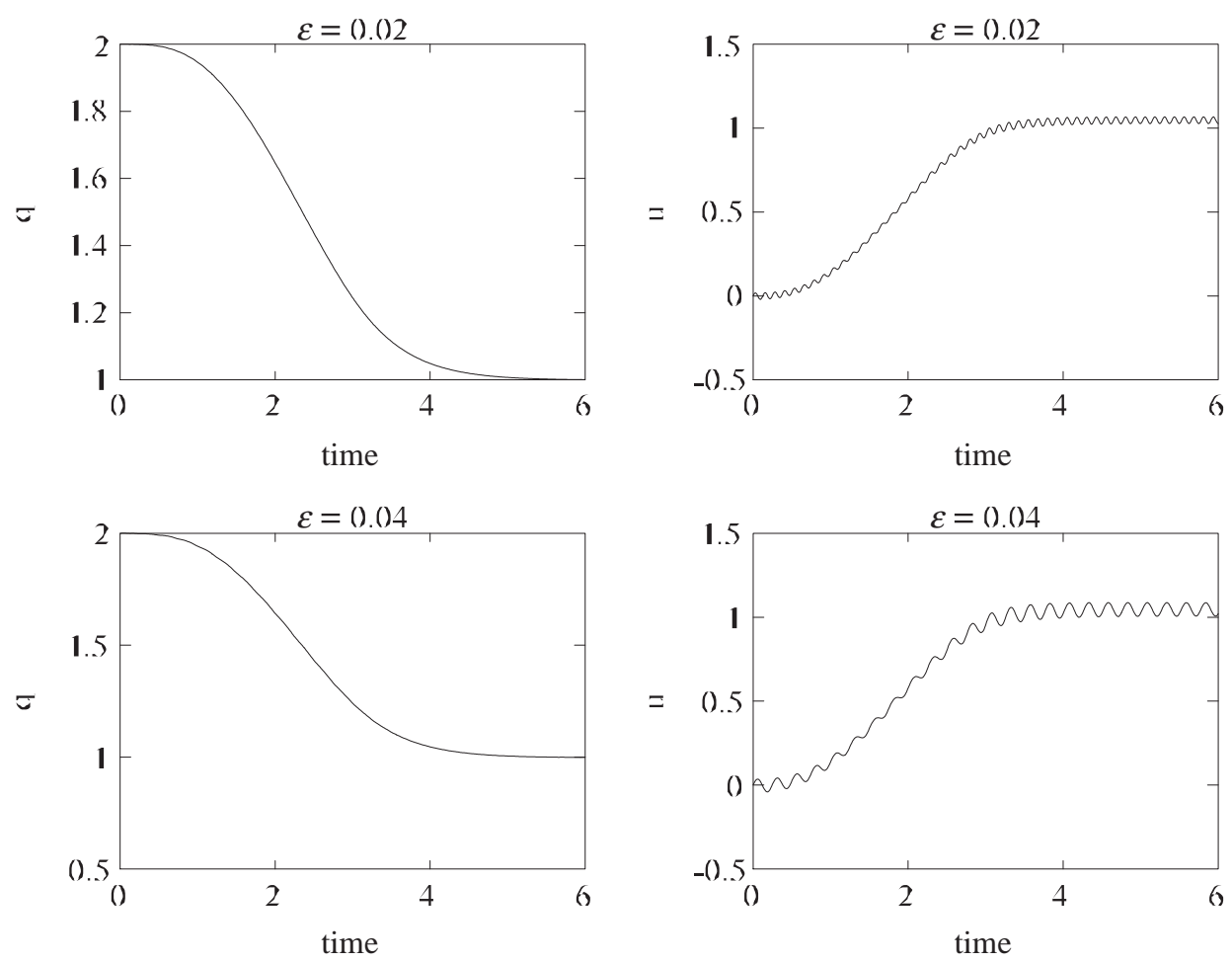

Fig. 2. High-frequency control for the variable-length pendulum

Remark 7. The two other non-flat examples of [26], namely the simple and the double Kapitsa pendulums, are also made flat by employing similar highfrequency controls. This strategy will be completely formalized elsewhere. Note that the other averaged systems considered in [26] are linearizable by non trivial, i.e., non static, dynamic feedback, contrarily to our simpler present situation.

5. Conclusion. Differential algebra is certainly not the only possible language for investigating flatness. Jakubczyk [36, 37] has proposed an extension of this formalism by utilizing differential rings of smooth or analytic functions (see [48, 39] for a discussion concerning differential algebra in a broader context). It has been recently demonstrated by the authors $[25,24]$ that a differential geometric setting is very natural (see also [47]). It is based on Lie-Bäcklund mappings between diffieties, i.e., on mappings between some infinite-dimensional Fréchet manifolds equipped with one-dimensional Cartan distributions (cf. [58]). 


\section{References}

[1] J. Baillieul, Stable average motions of mechanical systems subject to periodic forcing, reprint, 1993.

[2] J. Bentsman, Vibrational control of a class of nonlinear multiplicative vibrations, IEEE Trans. Automat. Control 32 (1987), 711-716.

[3] A. Bressan and F. Rampazzo, On differential systems with quadratic impulses and their applications to Lagrangian mechanics, SIAM J. Control Optim. 31 (1993), 12051220 .

[4] E. Cartan, Sur l'intégration de certains systèmes indéterminés d'équations différentielles, J. Reine Angew. Math. 145 (1915), 86-91; also in: Oeuvres Complètes, part II, vol. 2, CNRS, Paris, 1984, 1164-1174.

[5] P. J. Cassidy, Differential algebraic groups, Amer. J. Math. 94 (1972), 891-954.

[6] B. Charlet, J. Lévine and R. Marino, On dynamic feedback linearization, Systems Control Letters 13 (1989), 143-151.

[7] - - - -, Sufficient conditions for dynamic state feedback linearization, SIAM J. Control Optim. 29 (1991), 38-57.

[8] D. Claude, Everything you always wanted to know about linearization, in: M. Fliess and M. Hazewinkel (ed.), Algebraic and Geometric Methods in Nonlinear Control Theory, Reidel, Dordrecht, 1986, 181-226.

[9] P. M. Cohn, Free Rings and their Relations, 2nd ed., Academic Press, London, 1985.

[10] J. M. Coron, Linearized control systems and applications to smooth stabilization, SIAM J. Control Optim. 1994.

[11] E. Delaleau et M. Fliess, Algorithme de structure, filtrations et découplage, C.R. Acad. Sci. Paris Sér. I 315 (1992), 101-106.

[12] M. D. Di Benedetto, J. W. Grizzle and C. H. Moog, Rank invariants of nonlinear systems, SIAM J. Control Optim. 27 (1989), 658-672.

[13] S. Diop, Elimination in control theory, Math. Control Signals Systems 4 (1991), 17-32.

[14] - Differential-algebraic decision methods and some applications to system theory, Theoret. Comput. Sci. 98 (1992), 137-161.

[15] M. Fliess, Automatique et corps différentiels, Forum Math. 1 (1989), 227-238.

[16] - Generalized controller canonical forms for linear and nonlinear dynamics, IEEE Trans. Automat. Control 35 (1990), 994-1001.

[17] - Some basic structural properties of generalized linear systems, Systems Control Letters 15 (1990), 391-396.

[18] - , A remark on Willems' trajectory characterization of linear controllability, ibid. 19 (1992), 43-45.

[19] M. Fliess and S. T. Glad, An algebraic approach to linear and nonlinear control, in: H. J. Trentelman and J. C. Willems (eds.), Essays on Control: Perspectives in the Theory and its Applications, Birkhäuser, Boston, 1993, 223-267.

[20] M. Fliess and M. Hasler, Questioning the classical state space description via circuit examples, in: M. A. Kashoek, J. H. van Schuppen, and A. C. M. Ran (eds.), Realization and Modelling in System Theory, MTNS'89, volume I, Birkhäuser, Boston, 1990, 1-12.

[21] M. Fliess, J. Lévine, P. Martin and P. Rouchon, On differentially flat nonlinear systems, in: Proc. IFAC-Symposium NOLCOS'92, Bordeaux, 1992, 408-412.

[22] - , - - - - Sur les systèmes non linéaires différentiellement plats, C. R. Acad. Sci. Paris Sér. I 315 (1992), 619-624.

[23] - - - - - - Défaut d'un système non linéaire et commande haute fréquence, ibid. 316 (1993), 513-518.

[24] - - - - - , Linéarisation par bouclage dynamique et transformations de Lie-Bäcklund, ibid. 317 (1993), 981-986. 
[25] M. Fliess, J. Lévine, P. Martin and P. Rouchon, Towards a new differential geometric setting in nonlinear control, in: Proc. Internat. Geometric Coll., Moscow, May 1993.

[26] - , - - - - Flatness and defect of nonlinear systems: introductory theory and examples, Internat. J. Control, 1995.

[27] M. Fliess, J. Lévine and P. Rouchon, A simplified approach of crane control via a generalized state-space model, in: Proc. 30th IEEE Control Decision Conf., Brighton, 1991, 736-741.

[28] - , - - - A generalized state variable representation for a simplified crane description, Internat. J. Control 58 (1993), 277-283.

[29] J. Guckenheimer and P. Holmes, Nonlinear Oscillations, Dynamical Systems and Bifurcations of Vector Fields, Springer, New York, 1983.

[30] R. Hartshorne, Algebraic Geometry, Springer, New York, 1977.

[31] D. Hilbert, Über den Begriff der Klasse von Differentialgleichungen, Math. Ann. 73 (1912), 95-108; also in: Gesammelte Abhandlungen, Vol. III, Chelsea, New York, 1965, 81-93.

[32] A. Isidori, Control of nonlinear systems via dynamic state feedback, in: M. Fliess and M. Hazewinkel (eds.), Algebraic and Geometric Methods in Nonlinear Control Theory, Reidel, 1986.

[33] A. Isidori, Nonlinear Control Systems, 2nd ed., Springer, New York, 1989.

[34] A. Isidori, C. H. Moog, and A. De Luca, A sufficient condition for full linearization via dynamic state feedback, in: Proc. 25th IEEE Conf. Decision Control, 1986, 203-208.

[35] N. Jacobson, Basic Algebra, I and II, 2nd ed., Freeman, New York, 1985.

[36] B. Jakubczyk, Remarks on equivalence and linearization of nonlinear systems, in: Proc. IFAC-Symposium NOLCOS'92, Bordeaux, 1992, 393-397.

[37] - Invariants of dynamic feedback and free systems, in: Proc. ECC'93, Groningen, 1993, $1510-1513$.

[38] J. Johnson, Kähler differentials and differential algebra, Ann. of Math. 89 (1969), 92-98.

[39] - Order for systems of differential equations and a generalization of the notion of differential ring, J. Algebra 78 (1982), 91-119.

[40] T. Kailath, Linear Systems, Prentice-Hall, Englewood Cliffs, N.J., 1980.

[41] E. R. Kolchin, Differential Algebra and Algebraic Groups, Academic Press, New York, 1973.

[42] -, Differential Algebraic Groups, Academic Press, Orlando, 1985.

[43] C.-W. Li and Y.-K. Feng, Functional reproducibility of general multivariable analytic nonlinear systems, Internat. J. Control 45 (1987), 255-268.

[44] P. Martin, Contribution à l'étude des systèmes diffèrentiellement plats, $\mathrm{PhD}$ thesis, École des Mines de Paris, 1992.

[45] S. M. Meerkov, Principle of vibrational control: theory and applications, IEEE Trans. Automat. Control 25 (1980), 755-762.

[46] H. Nijmeijer and A. J. van der Schaft, Nonlinear Dynamical Control Systems, Springer, New York, 1990.

[47] J. B. Pomet, A differential geometric setting for dynamic equivalence and dynamic linearization, this volume, 319-339.

[48] J. F. Ritt, Systems of differential equations i. theory of ideals, Amer. J. Math. 60 (1938), $535-548$.

[49] -, Differential Algebra, Amer. Math. Soc., New York, 1950.

[50] A. Seidenberg, Some basic theorems in differential algebra (characteristic p, arbitrary), Trans. Amer. Math. Soc. 73 (1952), 174-190.

[51] W. F. Shadwick, Absolute equivalence and dynamic feedback linearization, Systems Control Letters 15 (1990), 35-39. 
[52] E. D. Sontag, Finite dimensional open loop control generator for nonlinear control systems, Internat. J. Control 47 (1988), 537-556.

[53] —, Universal nonsingular controls, Systems Control Letters 19 (1992), 221-224.

[54] H. J. Sussmann and V. Jurdjevic, Controllability of nonlinear systems, J. Differential Equations 12 (1972), 95-116.

[55] H. J. Sussmann and W. Liu, Limits of highly oscillatory controls and the approximation of general paths by admissible trajectories, in: Proc. 30th IEEE Control Decision Conf., Brighton, 1991, 437-442.

[56] J. C. Willems, Paradigms and puzzles in the theory of dynamical systems, IEEE Trans. Automat. Control 36 (1991), 259-294.

[57] D. J. Winter, The Structure of Fields, Springer, New York, 1974.

[58] V. V. Zharinov, Geometrical Aspect of Partial Differential Equations, World Scientific, Singapore, 1992. 